\title{
Article
}

\section{Demonstration of the Quantum No-Hiding Theorem in a Category Theoretic Framework: An IBM Quantum Experience}

\author{
Amolak Ratan Kalra ${ }^{1}$ (D), Shiroman Prakash ${ }^{2}$, Bikash K. Behera ${ }^{3}$ and Prasanta K. Panigrahi ${ }^{4, *}$ \\ 1 Department of Electrical Engineering, Dayalbagh Educational Institute, Dayalbagh, Agra 282005, India; \\ amolakratankalra@gmail.com \\ 2 Department of Physics and Computer Science, Dayalbagh Educational Institute, Dayalbagh, Agra 282005, \\ India; shiroman@gmail.com \\ 3 Department of Physical Sciences, Indian Institute of Science Education and Research Kolkata, Mohanpur \\ 741246, West Bengal, India; bkb13ms061@iiserkol.ac.in \\ 4 Department of Physical Sciences, Indian Institute of Science Education and Research Kolkata, Mohanpur \\ 741246, West Bengal, India \\ * Correspondence: pprasanta@iiserkol.ac.in; Tel.: +91-974-891-8201
}

\begin{abstract}
In this note, we demonstrate the quantum no-hiding theorem of Braunstein and Pati [Phys. Rev. Lett. 98, 080502 (2007)] using the IBM 5Q quantum processor. We also analyze the circuit using the ZX calculus of Coecke and Duncan [New J Phys. 13(4), 043016 (2011)], which provides a pictorial/category-theoretic demonstration of the no-hiding theorem.
\end{abstract}

Keywords: IBM quantum experience; no-hiding theorem; quantum information

\section{1. introduction}

IBM has developed a 5-qubit, SQUID-based quantum computer, ibmqx2, which has been released to the research community at large via a web-based interface called IBM Quantum Experience [1] (IBM QE). It is world's first commercial quantum computing service provided by IBM, and permits a user to run quantum algorithms via the IBM cloud and implement quantum circuits. Using this web interface researchers have run a variety of quantum computing experiments and demonstrations, e.g., [2-11].

Two well-known and important qualitative features of quantum information are that, unlike classical information, it cannot be cloned [12] or deleted [13]. A closely-related, and equally important feature of quantum information, proven by Braunstein and Pati [14] is that quantum information also cannot be "hidden", in a sense that we will make precise in Section 1.1. The quantum no-hiding theorem is relevant questions about thermalization in quantum systems [15], the black hole information loss paradox, and other areas in quantum information where state randomization plays a role, e.g. [16].

In this note, we use IBM Quantum Experience to test and demonstrate the quantum no-hiding theorem. We remark that this work is inspired by the first experimental test of no-hiding theorem using an NMR based quantum information system [17].

\subsection{Review of The Quantum No-Hiding Theorem}

In classical physics, it is possible to "hide" information in correlations. A simple example is the one-time pad [18], used in cryptography. Consider a message which is a binary string: $M=$ $M_{1} M_{2} M_{3} \ldots$, and a secret key which is also binary string of the same length: $K=K_{1} K_{2} K_{3} \ldots$ Create an encoded message $M^{\prime}$ by performing a bitwise XOR of $K$ on $M$ (i.e., flip the $M_{i}$ if $K_{i}=1$, otherwise leave $M_{i}$ unchanged.) Shannon proved that an agent with access to only the encoded string $M^{\prime}$, and not the key $K$, has no information about the original message $M$ [19]. Of course, an agent with access to only the key $K$, and not the encoded string $M^{\prime}$, also has no information about the original message $M$. To obtain any information about the original message $M$ an agent requires at least some information about the correlations between the key $K$ and the encoded string $M^{\prime}$. In this sense, one can say that the 


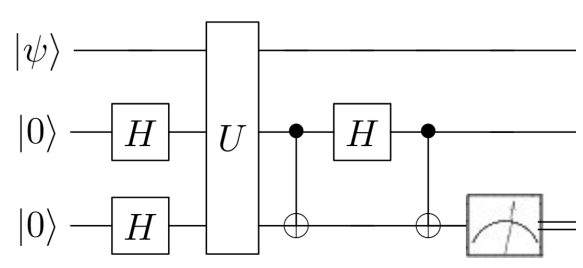

Figure 1. Circuit demonstrating no-hiding theorem.

information originally contained in $M$ has been hidden in correlations between the encoded string $M^{\prime}$ and the key $K$.

Braunstein and Pati proved that, in quantum mechanics, hiding information in such a way is impossible [14].

Specifically, let us define a bleaching process as a process which transforms the state of a quantum system $M$ to the maximally mixed state (or, more generally, any fixed density matrix $\rho$ ), regardless of what the initial state of the system was. After bleaching, an agent with access to only the system $M$ has no information about its original state. Bleaching processes are clearly not unitary, but can take place if $M$ is an open quantum system coupled to an environment.

After bleaching, the quantum information previously contained in $M$ cannot be truly lost (assuming the universe is a closed quantum system), so it is natural to ask where in the universe it resides. The no-hiding theorem states that, in any bleaching process, the quantum information must be transferred completely to the environment, and (unlike the example of the classical one-time pad) cannot reside within correlations between the environment and the original system $M$. In particular, an agent who has access to only the environment and not the bleached system $M$, can, in principle, always completely recover the quantum information initially stored in $M$, via an appropriate unitary transformation.

For a large environment, this unitary transformation may be hopelessly complicated. However, for a smaller "environment" consisting of a few qubits, it is possible to explicitly construct the required unitary transformation and thereby directly demonstrate the no-hiding theorem, as we show below.

\section{Circuit to Demonstrate the No-Hiding Theorem}

The circuit we use to demonstrate the no-hiding theorem is depicted in Figure 1.

In this circuit, the system $M$ to be bleached is a single qubit. In order to bleach one qubit, we require an "environment" consisting of at least two ancilla qubits. A 3-qubit unitary "randomization" operator $\mathbf{U}$ that achieves bleaching is the controlled operation that applies one of the operators four Pauli operators $I$ (the identity), $\mathbf{X}, \mathbf{Y}$ or $\mathbf{Z}$ to $M$, depending on whether the two ancilla qubits are in the state $|00\rangle,|01\rangle,|10\rangle$ or $|11\rangle$ respectively. We can write this as

$$
\mathbf{U}=\mathbb{1} \otimes|00\rangle\langle 00|+\mathbf{X} \otimes| 01\rangle\langle 01|+i \mathbf{Y} \otimes| 10\rangle\langle 10|+\mathbf{Z} \otimes| 11\rangle\langle 11| .
$$

Let the state of $M$ initially be $|\psi\rangle$. Suppose we initialize the two ancilla qubits in the state

$$
\frac{1}{4}(|00\rangle+|01\rangle+|10\rangle+|11\rangle) .
$$

Then after applying the unitary randomization operator $\mathbf{U}$, and tracing out the ancilla qubits, it is easy to see that the state of $M$ is the maximally mixed state, regardless of its initial state $|\psi\rangle$. Hence, we have bleached $M$.

The no-hiding theorem states that we can recover $|\psi\rangle$, acting exclusively on the two ancillas. As mentioned earlier, for a larger environment, and a complicated randomization operator $\mathbf{U}$, this might be an intractable task, but for our relatively small system, it is straightforward to to determine the 


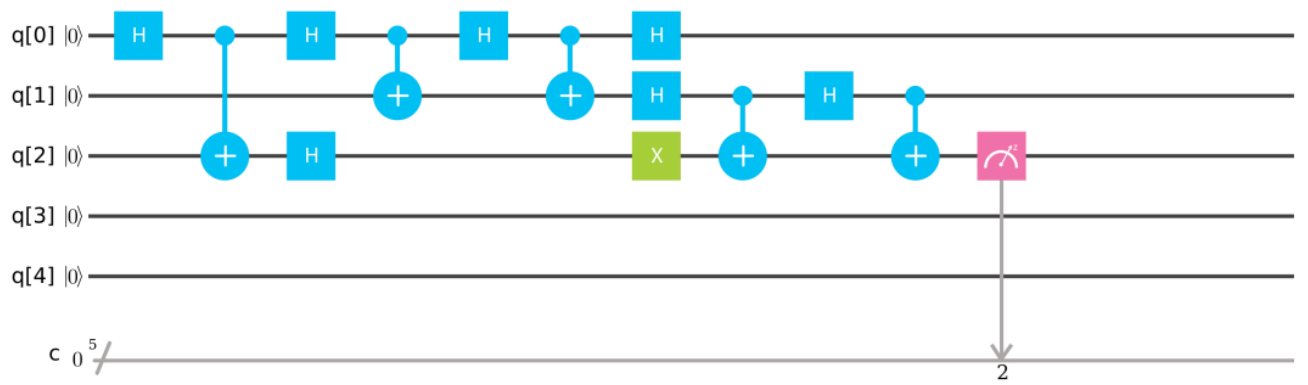

Figure 2. IBM quantum circuit illustrating no-hiding theorem.

two-qubit unitary that decodes the apparently lost information from the environment. We do this using the pictorial XZ calculus of Coecke and Duncan [20] in Section 4.

The resulting circuit, which includes initialization, bleaching and decoding, is shown below.

\section{IBM QE Implementation}

We implemented the circuit including the unitary operator on IBM QE. It is straightforward to see that the randomization operator $\mathbf{U}$ can be realized as a sequence of controlled-NOT and controlled-Z gates, as shown in Figure 3.

Let the initial state of the qubit $q[0]$ in Figure 3 be $|\psi\rangle$. To demonstrate the no-hiding theorem, we need to verify that, after applying the bleaching operation, the qubit $q[0]$ is in the maximally mixed state, and the qubit $q[2]$ is in the state $|\psi\rangle$.

We do this by state tomography - we repeat the experiment many times (1024 shots), measuring one of the three Pauli-matrices in each round, to determine $\langle X\rangle,\langle Y\rangle$ and $\langle Z\rangle$, from which we can reconstruct the experimentally obtained density matrix $\rho^{(\mathrm{E})}$ as follows:

$$
\rho^{(\mathrm{E})}=\frac{1}{2}(I+\langle X\rangle X+\langle Y\rangle Y+\langle Z\rangle Z)
$$

We compare to the theoretical density matrix:

$$
\rho^{(\mathrm{T})}=|\Psi\rangle\langle\Psi|
$$

Note that IBM quantum experience also allows us to simulate the results of the quantum computation. We use the notation $\rho^{(\mathrm{S})}$ to denote density matrices obtained by simulation.

We carried out the experiment for a variety of states $|\psi\rangle$, of the form $|\psi\rangle=\cos \theta|0\rangle+\sin \theta|1\rangle$ and found good agreement with expected results. Let us present the results explicitly when $\theta=\frac{\pi}{8}$. Then, for the state of the qubit $q[2]$, we expect

$$
\rho_{q}^{(\mathrm{T})}=\left[\begin{array}{ll}
0.860 & 0.346 \\
0.346 & 0.140
\end{array}\right]
$$

and we obtained the following results:

$$
\begin{gathered}
\rho_{q}^{(\mathrm{E})}=\left[\begin{array}{ll}
0.792 & 0.331 \\
0.331 & 0.208
\end{array}\right]+i\left[\begin{array}{cc}
0.000 & 0.009 \\
-0.009 & 0.000
\end{array}\right] \\
\rho_{q}^{(\mathrm{S})}=\left[\begin{array}{ll}
0.860 & 0.340 \\
0.340 & 0.140
\end{array}\right]+i\left[\begin{array}{cc}
0.000 & -0.09 \\
0.09 & 0.000
\end{array}\right]
\end{gathered}
$$




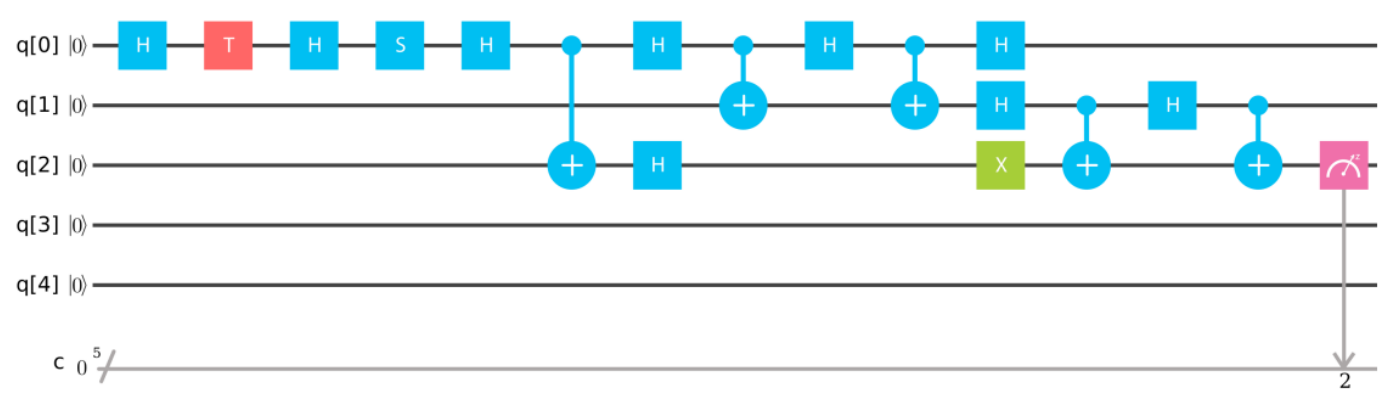

Figure 3. IBM quantum circuit illustrating no-hiding theorem with $\theta=\frac{\pi}{8}$

The number of shots used during this experiment was 1024 shots. These results are depicted graphically in Figure 4.

\section{Diagrammatic Derivation}

Here, we analyze the randomization circuit presented above using the ZX-calculus [20]. See [20] for details of the notation, which can be understood either as a convenient set of pictorial rules for demonstrating the equivalence of certain quantum circuits, or as an alternative axiomatization of quantum mechanics using the framework of dagger symmetric monoidal categories. 
(1a)

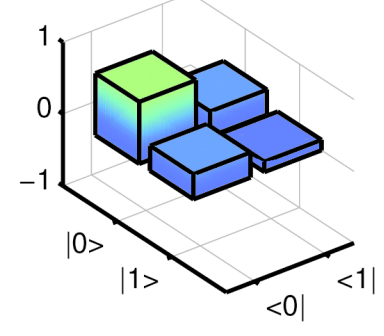

(1c)

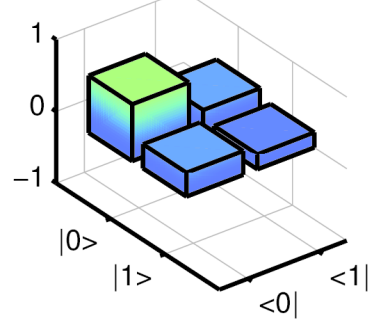

(1e)

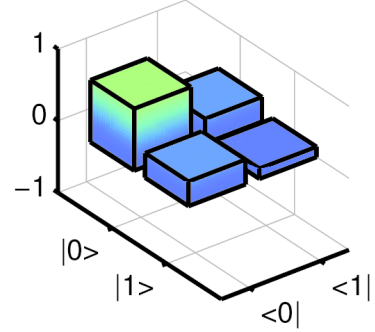

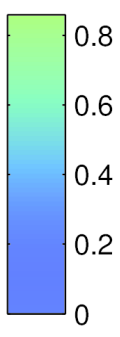

(1b)
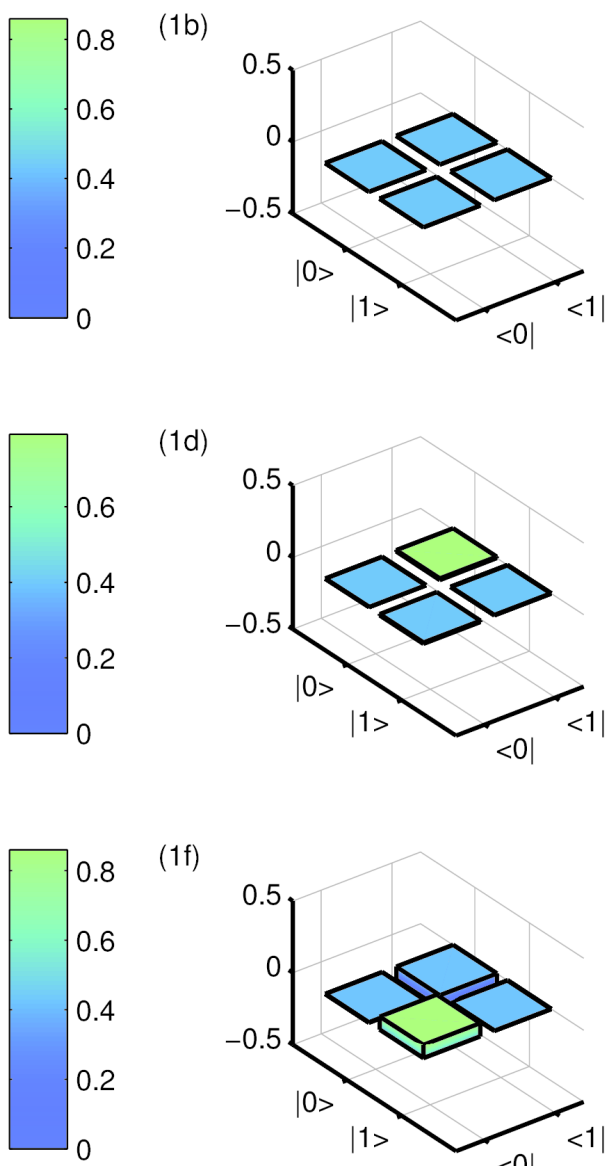

(1d)

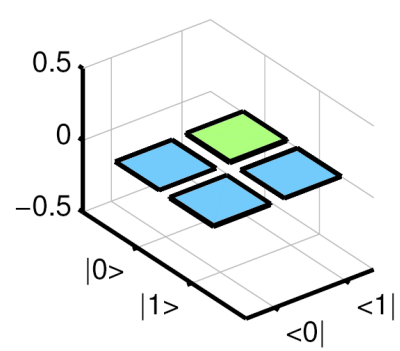

(1f)

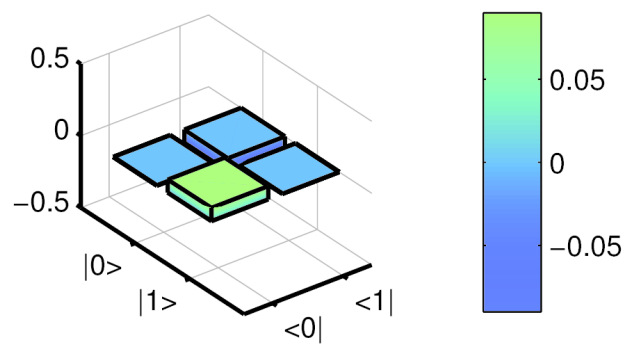

Figure 4. Depicting Real (left) and imaginary (right) parts of the reconstructed theoretical (1a,1b), run $(1 \mathrm{c}, 1 \mathrm{~d})$ and simulated $(1 \mathrm{e}, 1 \mathrm{f})$ density matrices. 

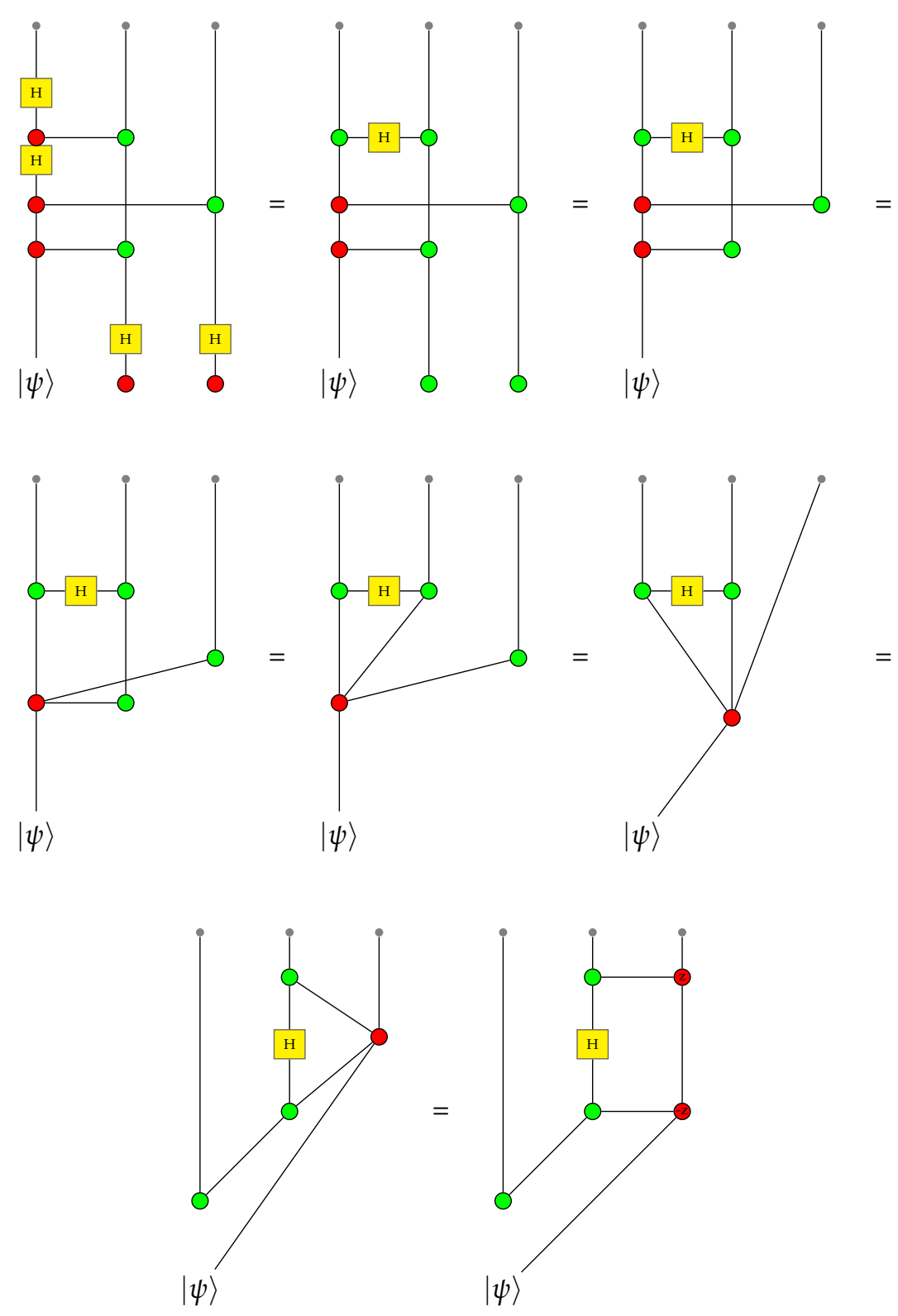

These diagrams allow us to trace the flow of quantum information from the original system to the ancillas. We see that the state $|\psi\rangle$ is encoded in the two ancilla qubits, while the first qubit now contains no information about $|\psi\rangle$. From the analysis, we also see that by applying the $\operatorname{CNOT}(2,3)$, Hadamard(2), $\operatorname{CNOT}(2,3)$ gates, we can decode the state $|\psi\rangle$ from the two ancilla qubits. The above figures were created using the software Quantomatic [21].

While the above derivation demonstrates the flow of information, it would be very interesting to provide a purely diagrammatic (or category-theoretic) proof of the no-hiding theorem, perhaps similar in spirit to the category theoretic versions of the no-cloning theorem [22]. It would also be very educational to understand the no-hiding theorem in the context of the systems-theoretic framework of [23], where quantum information acts as a "through" variable, analogous to current in an electrical circuit, and obeys an analogue of Kirchoff's current law.

\section{Conclusions}

To conclude, we have demonstrated here the experimental verification of the no-hiding theorem using IBM QE. ZX calculus has been utilized to simplify the circuit required for illustrating this theorem. 
Quantum State Tomography has been performed to check the accuracy of the implementation. A number of directions can be pursued in future. One of them is the extension of the no-hiding theorem to imperfect hiding processes. We can also investigate the applications of this theorem for retrieving information from a noisy environment. In future, no-hiding theorem can also be explicated by using highly entangled states, such as Brown et al. state [24].

\section{Acknowledgements}

We are extremely grateful to the IBM team and the IBM QE project. The discussions in this paper do not reflect any opinion of the IBM QE team or IBM. ARK thanks IISER Kolkata for hospitality during which part of this work was completed. ARK will also like to thank the National Initiative for Undergraduate Science (NIUS) Physics. SP thanks ICTS, Bengaluru for hospitality, and acknowledges the support of a DST INSPIRE Faculty Award. BKB acknowledges DST Inspire Fellowship.

\section{References}

1. IBM Q. Available online: URL http:/ / research.ibm.com/ibm-q/ (accessed on 27th Sept. 2017).

2. Behera, B.K.; Banerjee, A.; Panigrahi, P.K. Experimental realization of quantum cheque using a five-qubit quantum computer. 2017, arXiv:1707.00182.

3. Sisodia, M.; Verma, V.; Thapliyal, K.; Pathak, A. Teleportation of a qubit using entangled non-orthogonal states: a comparative study. Quantum Inf. Process. 2017, 16, DOI: 10.1007/s11128-017-1526-x.

4. Sisodia, M.; Shukla, A.; Pathak, A. Experimental realization of nondestructive discrimination of Bell states using a five-qubit quantum computer. 2017, arXiv:1705.00670.

5. Sisodia, M.; Shukla, A.; Thapliyal, K.; Pathak, A. Design and experimental realization of an optimal scheme for teleportion of an n-qubit quantum state. 2017, arXiv:1704.05294.

6. Huffman, E.; Mizel, A. Violation of noninvasive macrorealism by a superconducting qubit: Implementation of a Leggett-Garg test that addresses the clumsiness loophole. Phys. Rev. A 2017, 95, 032131, DOI: 10.1103/PhysRevA.95.032131.

7. Alsina, D.; Latorre, J. Experimental test of Mermin inequalities on a five-qubit quantum computer. Phys. Rev. A 2016, 94, 012314, DOI: 10.1103/PhysRevA.94.012314.

8. Berta, M.; Wehner, S.; Wilde, M.M. Entropic uncertainty and measurement reversibility. New J. Phys. 2016, 18, 073004, DOI: 10.1088/1367-2630/18/7/073004.

9. Devitt, S.J. Performing quantum computing experiments in the cloud. Phys. Rev. A 2016, 94, 032329, DOI: 10.1103/PhysRevA.94.032329.

10. Linke, N.M.; Maslov, D.; Roetteler, M.; Debnath, S.; Figgatt, C.; Landsman, K.A.; Wright, K.; Monroe, C. Experimental comparison of two quantum computing architectures. Proc. Natl. Acad. Sci. 2017, 114, 3305-3310, DOI: 10.1073/pnas.1618020114.

11. Wootton, J.R. Demonstrating non-Abelian braiding of surface code defects in a five qubit experiment. Quantum Sci. Technol. 2017, 2, 015006, DOI: 10.1088/2058-9565/aa5c73.

12. Wootters, W.K.; Zurek, W.H. A single quantum cannot be cloned. Nature 1982, 299, 802, DOI: 10.1038/299802a0.

13. Pati, A.K.; Braunstein, S.L. Impossibility of deleting an unknown quantum state. Nature 2000, 404, 164, DOI: 10.1038/404130b0.

14. Braunstein, S.L.; Pati, A.K. Quantum Information Cannot Be Completely Hidden in Correlations: Implications for the Black-Hole Information Paradox. Phys. Rev. Lett. 2007, 98, 080502, DOI: 10.1103/PhysRevLett.98.080502.

15. Popescu, S.; Short, A.J.; Winter, A. Entanglement and the foundations of statistical mechanics. Nat Phys. 2006, 2, 754, DOI: 10.1038/nphys444.

16. Ambainis, A.; Mosca, M.; Tapp, A; Wolf, R.D. Private quantum channels. In Fndns. of Computer Sci. 2000. Proceedings. 41st Annual Symposium on, pages 547-553, 2000.

17. Samal, J.R.; Pati, A.K.; Kumar, A. Experimental Test of the Quantum No-Hiding Theorem. Phys. Rev. Lett. 2011, 080401, DOI: 10.1103/PhysRevLett.106.080401. 
18. Vernam, F.S. Cipher Printing Telegraph Systems For Secret Wire and Radio Telegraphic Communications. Trans. American Inst. Elec. Eng. 1926, 45, 295, DOI: 10.1109/T-AIEE.1926.5061224.

19. Shannon, C.E. Communication theory of secrecy systems. Bell Labs Tech. J. 1949, 28, 656, DOI: 10.1002/j.1538-7305.1949.tb00928.x.

20. Coecke, B.; Duncan, R. Interacting quantum observables: categorical algebra and diagrammatics, New J. Phys. 2011, 13, 043016, DOI: 10.1088/1367-2630/13/4/043016.

21. Kissinger, A.; Zamdzhiev A. Quantomatic: A Proof Assistant for Diagrammatic Reasoning. Spring. Internatl. Publish. 2015, 326, DOI: 10.1007/978-3-319-21401-6_22.

22. Abramsky S. No-cloning in categorical quantum mechanics. 2012, arXiv:0910.2401v2.

23. Srivastava, D.P.; Sahni, V.; Satsangi, P.S. Graph-theoretic quantum system modelling for information/computation processing circuits. Internatl. J. Gen. Sys. 2011, 40, 777, DOI: 10.1080/03081079.2011.602016.

24. Brown, I.D.K.; Stepney, S.; Sudbery, A.; Braunstein, S.L. Searching for highly entangled multi-qubit states. J. Phys. A: Math. Gen. 2005, 38, DOI: 10.1088/0305-4470/38/5/013. 